MAGNETOHYDRODYNAMICS Vol. 54 (2018), No. 1-2, pp. 85-90

DOI: $10.22364 / \mathrm{mhd} .54 .1-2.15$

\title{
PARTICLE AGGREGATION IN MAGNETIC FLUID LAYER IN ELECTRIC FIELD
}

\author{
Yu.A. Larionov, V.M. Kozhevnikov, I.Yu. Chuenkova, A.A. Antonova \\ North-Caucasus Federal University, 1 Pushkin str. 355009, Stavropol, Russia \\ e-Mail: chuenkova@gmail.com
}

The action of a DC electric field directed perpendicularly to an initially homogeneous magnetic fluid (MF) layer gives rise to a new, more concentrated phase, i.e. microdrop formations in the near-electrode layer at a threshold field. Despite of the thorough enough studies [2-8], the fundamental understanding of the structure formation and near-electrode layer properties is still the question of debate.

Introduction. The action of a DC electric field directed perpendicularly to an initially homogeneous magnetic fluid (MF) layer gives rise to a new, more concentrated phase, i.e. microdrop formations in the near-electrode layer (Fig. 1) at a threshold field $E_{\mathrm{C}}[1-3]$. The formation of microdrops is associated with the high-concentrated disperse particle zones due to their electrophoretic migration to the electrodes and also with the influence of the electric field on the aggregation stability of colloidal systems. The conductivity of the near-electrode MF layer is three orders of magnitude smaller than that of the volume $\left(10^{-9} \mathrm{Ohm}^{-1} \mathrm{~m}^{-1}\right)$ [4]. This can be associated with the adsorption of the surfactant on the electrodes. The surface charge density in the near-electrode layer is estimated as $4 \cdot 10^{-3} \mathrm{C} / \mathrm{m}^{2}[5]$. The solutions of oleic acid or butyl alcohol in kerosene do not form such an anomalously large charge. These near-electrode layers, differing in their properties from the MF in the volume, respond to the microstructure formation features, to the dependence of the threshold field on the layer thickness and to the MF conductivity. Despite of the thorough enough studies [2-8], the fundamental understanding of the structure formation and near-electrode layer properties is still the question of debate.

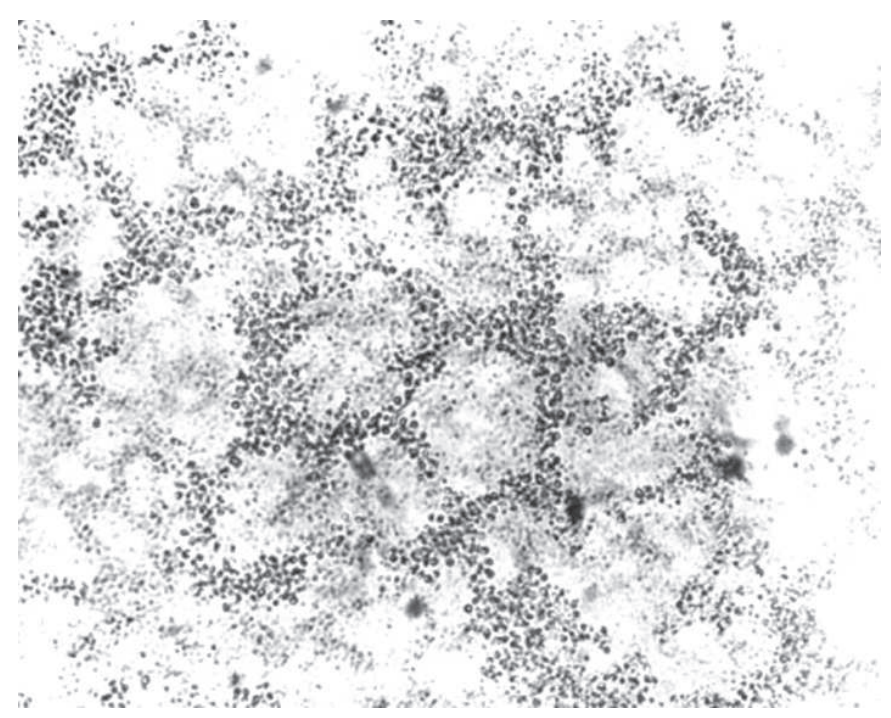

Fig. 1. Aggregated structure in the near-electrode layer. 
1. Experiment. A plane-parallel glass cell with an electrically conducting coating $\mathrm{In}_{2} \mathrm{O}_{3} \mathrm{SnO}_{2}$ had partially overlapping electrodes. A kerosene-based magnetic fluid with a solid phase concentration of 4-7\% formed a $15-50 \mu \mathrm{m}$ layer. Experimental methods for studying the structure formation included

i) an electrical method to register currents passing through the cell with the MF during the re-polarization process; the MF layer is located perpendicularly to the electric field created by DC and $\mathrm{AC}$ voltage sources with a tunable frequency $(0.1 \mathrm{~Hz} \ldots 1 \mathrm{MHz})$;

ii) visual observations of the structures in the near-electrode layer using a high-speed digital camera. To study the effect of the magnetic field, the MF cell was exposed to a uniform field created by Helmholtz coils.

2. Results and discussion. The structure formation process in the electric field has revealed some interesting features. One of the distinctive features of the emerging instability was the formation of micro-aggregates with periodic structures under the action of the electric field. The charged near-electrode layer had the shape of corrugated surface with finite amplitude. Clear tightly-packed corrugations were observed in the point source light (Fig. 2). The depth of the troughs was finite and increased with increasing field. Such restructuring in the MF layer caused by a differently charged surface was discussed in [9]. With the increase of the near-electrode layer corrugation, the homogeneity of the distribution of microdrop aggregates in the magnetic fluid was violated (the aggregates were assembled into a cellular structure (Fig. 1)).

A trough corrugation along the edges of the electrode at the final stage of instability was observed experimentally (Fig. 2). As noted in [9], the corrugation structure loses the energy of the cellular one. The corrugation structure is determined by the local conditions of the surface deformation along the electrode boundary, where the field is non-uniform. The experiment showed that troughs appeared along the boundaries and their period coincided with the dimension of the corrugation cells. Its structure was determined by the polarity of the lower electrode (Fig. 3). When it was positively charged, the troughs were aligned with the electrode edges and extended beyond the upper electrode, demonstrating energy advantages in charge distribution along the electrode boundary area. When it

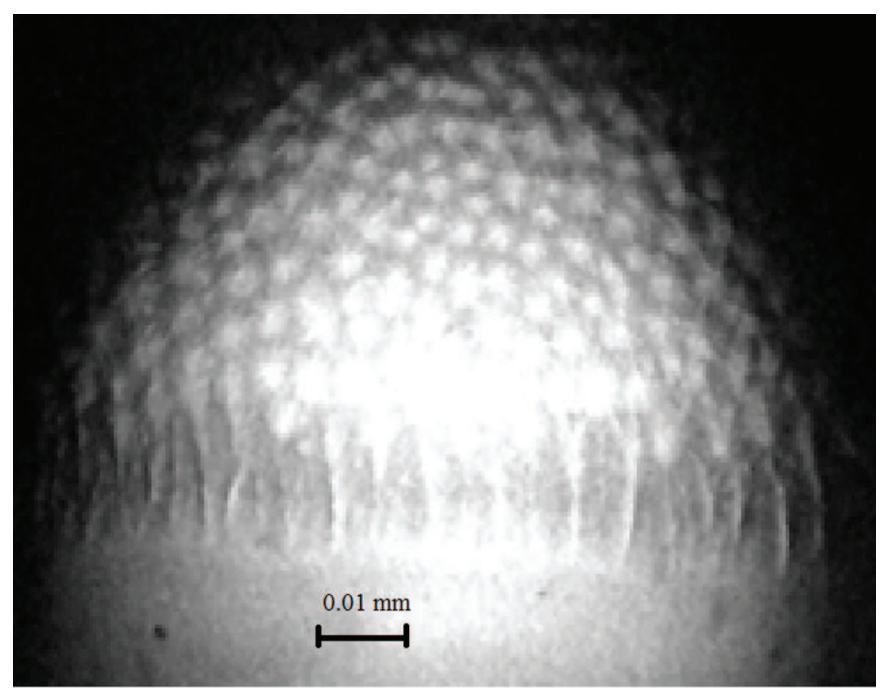

Fig. 2. The goffered boundary layer. 
Particle aggregation in magnetic fluid layer in electric field
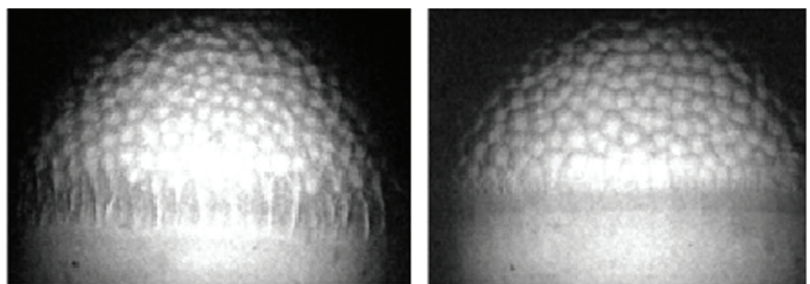

electrode -

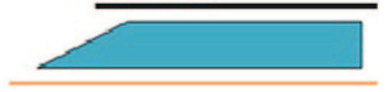

electrode +

electrode +
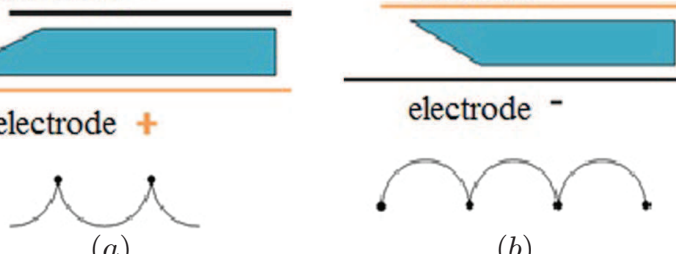

(b)

Fig. 3. A structured boundary layer at different polarity of the electrode $(U=7.5 \mathrm{~V})$; (a) anode top electrode, (b) cathode bottom electrode.

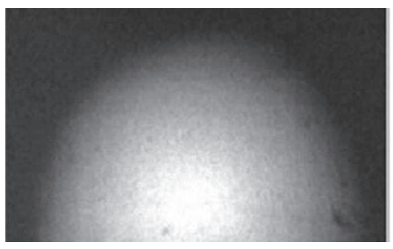

$U=6 \mathrm{~V}$

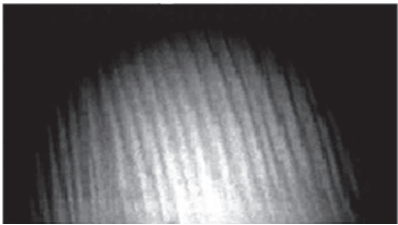

$U=9 \mathrm{~V}$

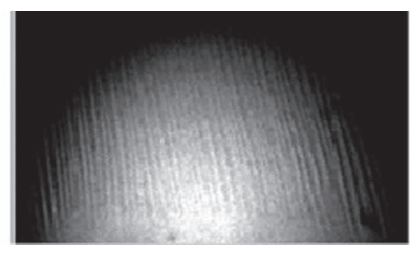

$U=7 \mathrm{~V}$

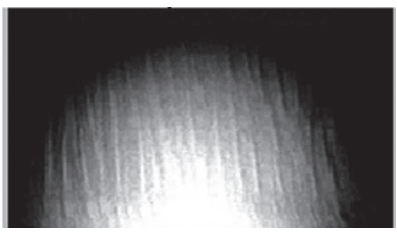

$U=12 \mathrm{~V}$

Fig. 4. A corrugated surface structure $(H=2 \mathrm{kA} / \mathrm{m})$.

was negatively charged, the troughs were located only inside the upper electrode, which means that the electrode and the interface are equally charged. As a result, the microdrops in the MF layer carrying a positive charge tended to locate mostly closer to the negative electrode. In an additional magnetic field directed along the electrode surface, the corrugation structure became groove-like, which is energetically favorable in the MF layer with a small surface tension. The structured layer changed the electrical polarity and was partially deformed if the charge sign of the electrode was changed.

The formation of the corrugated surface starts when the electric field intensity exceeds the threshold value $E_{\mathrm{C}}$. The corrugated structure is stable. The wave size of the corrugation depends on the intensity of the electric field and on the accumulated interface charge (Fig. 4).

Optical studies in scattered light have characterized the anisotropic properties of the near-surface layer. The explanation of optical anisotropy in the boundary layer by electrohydrodynamic flows at the electrode [10] contradicts to the earlier results [2], where the optical anisotropy outside the structured fluid under shear flow was ignored. 


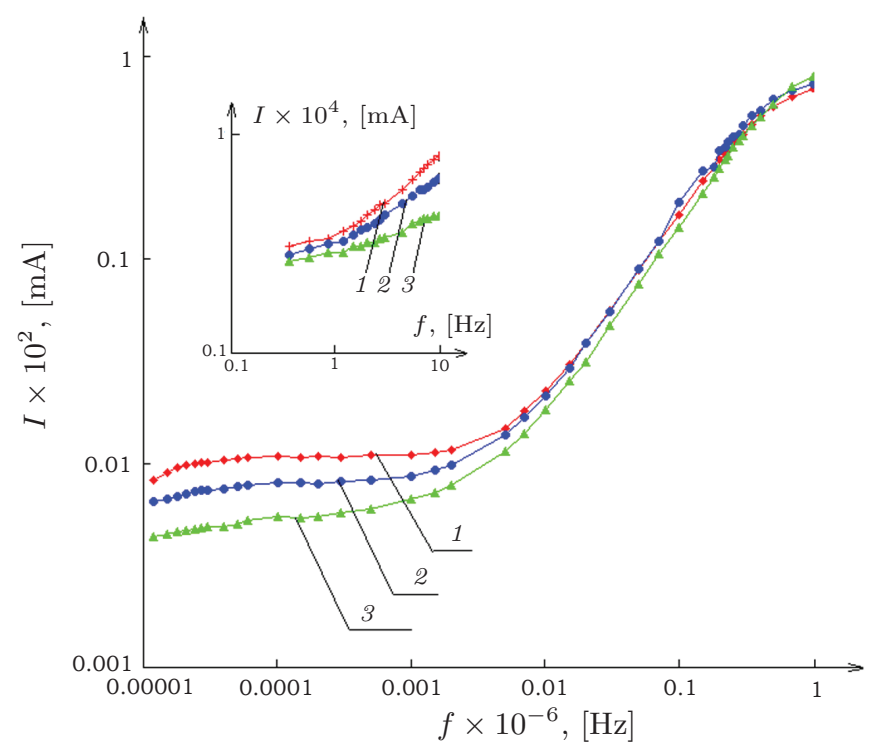

Fig. 5. Amplitude-frequency characteristics of a cell with an MF layer: 1 - sinusoidal voltage $U_{\mathrm{m}}=5 \mathrm{~V} ; 2-$ sinusoidal voltage $U_{\mathrm{m}}=5 \mathrm{~V}$ and polarizing voltage $U_{\text {pol }}=4 \mathrm{~V} ; 3-$ sinusoidal voltage $U_{\mathrm{m}}=4 \mathrm{~V}$ and polarizing voltage $U_{\mathrm{pol}}=5 \mathrm{~V}$.

The electric field alters the shape of the salvation shell of the magnetite particles and an induced charge forms. This enhances the particle interaction and some structures develop. As a result, the optical density of the layer increases.

New phases develop in the MF layer and coexist in the electric field in the following order. First, an anisotropic layer is formed. Its formation and destruction take much more time than the Maxwell-Wagner relaxation time for an MF with the conductivity $10^{-6} \mathrm{Ohm}^{-1} \mathrm{~m}^{-1}$. Then the concentrated drops with distinct borders are formed. They can also acquire a charge and participate in the electrophoretic motion. Microdrops are more frequently formed and destroyed in time than the anisotropic layer. The role of oleic acid in the observed processes is ambiguous.

Coalescence structures (static precipitation) are formed at last. The diversity of the structural phases affects the optical and electrical properties of the thin layer. One of them is the low coefficient of the interfacial tension at the structured layer boundary. The amplitude-frequency (AFC) and phase-frequency characteristics (PFC) of a cell with an MF layer reveal the phase changes in it (Figs. 5, 6).

The AC current, passing through the cell as an additional polarizing voltage, decreases. This can be explained as a dependence of the cell parameters (capacitance and resistance) on the polarizing voltage (Fig. 5). The phase shift between the current and the voltage is capacitive. The phase shift dependence on the frequency has two maxima: one at $20-30 \mathrm{~Hz}$, another at $50000-150000 \mathrm{~Hz}$ (Fig. 6).

At low frequencies, the current through the cell is non-sinusoidal. With increasing frequency up to $20 \mathrm{~Hz}$ its shape approaches a sine wave.

\section{Conclusions.}

1. The corrugated structure in the near-electrode magnetic fluid layer exposed to electric and magnetic fields is primarily formed due to the restructuring of the charged boundary surface. The dynamics of the changed boundary in the nearelectrode layer follows certain scenarios in accordance with external conditions.

2. The interface near the electrode in the cell with a magnetic fluid layer is 
Particle aggregation in magnetic fluid layer in electric field

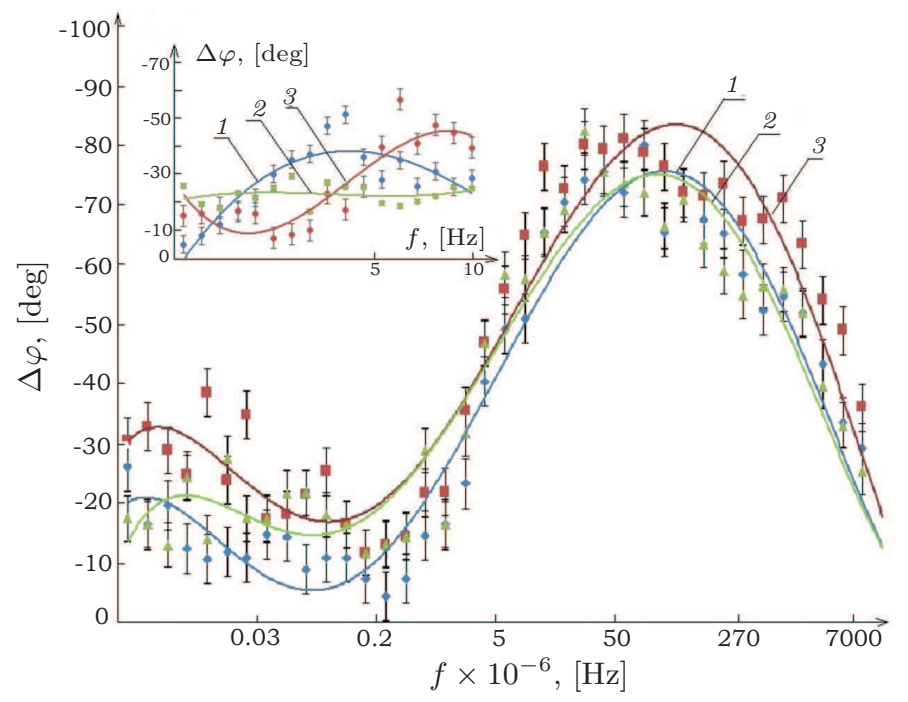

Fig. 6. Phase-frequency characteristics of a cell with an MF layer: 1 - sinusoidal voltage $U_{\mathrm{m}}=5 \mathrm{~V} ; 2-$ sinusoidal voltage $U_{\mathrm{m}}=4 \mathrm{~V}$ and polarizing voltage $U_{\mathrm{pol}}=5 \mathrm{~V} ; 3-$ sinusoidal voltage $U_{\mathrm{m}}=5 \mathrm{~V}$ and polarizing voltage $U_{\mathrm{pol}}=4 \mathrm{~V}$.

negatively charged, whereas the microdrops in the magnetic fluid layer are mostly positive-charged.

3. The observed phenomena provide the possibility of electrostatic and magnetic control of the structured MF layer.

\section{References}

[1] V.M. Kozhevnikov, Yu.A. Larionov, I.Yu. Chuenkova. Chuenkova. Dynamics of the boundary of a magnetic fluid in the near-electrode region. In: Proc. the 16th International Ples Scientific Conference on Nanodispersed Magnetics (Ivanovo, 2014), pp. 199-203 (in Russian).

[2] V.M. Kozhevnikov, Yu.A. Larionov, I.Yu. Chuenkova, M.I. Danilov. Obtaining the structured magnetic fluids in the electric field and their technical applications. Magnetohydrodynamics, vol. 40 (2004), no. 3, pp. 269-280.

[3] Yu.I. Dikansky, O.A. Nechaeva. Magnetic fluid structural transformations in electric and magnetic fields. Colloid Journal, vol. 65 (2003), no. 3, pp. 305-309.

[4] V.M. Kozhevnikov, Yu.A. Larionov, I.Yu. Chuenkova, T.F. MorozovA. Structural instability of a thin layer of a magnetic fluid in constant and alternating electric fields. In: Proc. the 15th International Ples Scientific Conference on Nanodisperse Magnetics (Ivanovo, 2012), pp. 99-103 (in Russian).

[5] V.V.Chekanov, E.A. Bondarenko, A.A. Hetmansky. Dynamics of formation and destruction of a layer of nanoparticles near an electrode in an electric field. Nanotechnics, vol. 17 (2009), no. 1, pp. 83-90 (in Russian). 
Yu.A. Larionov, V.M. Kozhevnikov, I.Yu. Chuenkova, A.A. Antonova

[6] T.F. Morozova, M.S. Demin. Analysis of the interaction between the processes of polarization and microstructuring in a magnetic fluid layer. Technical Physics, vol. 62 (2017), no. 2, pp. 314-321.

[7] V.M. Kozhevnikov, Yu.A. Larionov, M.S. Demin. Anomaly of amperetime characteristics of a thin layer of a magnetic fluid. In: Proc. the 14th International Ples Scientific Conference on Nanodispersed Magnetics (Ivanovo, 2010), pp. 218-223 (in Russian).

[8] K.V. ERIN. Formation and electro-optic investigation of the near-electrode space charge in colloidal solutions of magnetite in liquid dielectrics. Technical Physics, vol. 78 (2008), no. 4, pp.133-136.

[9] V.B. Shikin. Instability and reconstruction of a charged liquid surface. Advances in Physics, vol. 54 (2011), pp. 1203-1225.

[10] K.V. ERIN. Electrooptical effects in near-electrode layers in magnetic colloids based on liquid dielectrics. Optics and Spectroscopy, vol. 109 (2010), no. 3, pp. $498-503$.

Received 27.01.2012 